\title{
Maternal digital media use during infant feeding and the quality of feeding interactions
}

\author{
Alison K. Ventura*, Jordyn Levy, Sierra Sheeper \\ Department of Kinesiology and Public Health, California Polytechnic State University, San Luis Obispo, CA, USA
}

\section{A R T I C L E I N F O}

\section{Keywords:}

Breastfeeding

Digital media use

Mother-infant interaction

Infant feeding

\begin{abstract}
A B S T R A C T
Experimental research with parents of older children suggests parents' engagement with technological devices (e.g., television, mobile devices) in the presence of their children decreases the quality of parent-child interactions. Many mothers report frequent use of technological devices during infant feeding but, to date, few studies have explored the potential association between maternal technological device use during feeding and the quality of infant feeding interactions. To this end, mothers $(n=25)$ and their infants (mean age $=19.3 \pm 6.4$ weeks) participated in a within-subject, experimental study to explore associations between maternal digital media use and feeding interaction quality within a laboratory setting. Dyads were video-recorded while breastfeeding under two counterbalanced conditions: Digital Media Use versus Control. Mothers engaged their infants in significantly less cognitive growth fostering during the Digital Media Use compared to Control condition. Infants of mothers with typically low levels of technology use during feeding showed a significant decrease in their responsiveness to their mother during the Digital Media Use compared to Control condition. These results illustrate maternal digital media use was associated with decreases in some, but not all, aspects of the quality of the feeding interaction, meriting further investigation with larger, more diverse samples.
\end{abstract}

\section{Introduction}

When considering the types of caregiver-infant interactions that occur during early infancy, feeding interactions are central. Young infants typically feed 8-12 times per day (Fomon, 1993), meaning caregivers and infants spend a substantial portion of each day engaged in feeding. These interactions have both nutritional and social significance because high quality feeding interactions ensure the infant is properly nourished, but also afford the infant with opportunities for both socioemotional and cognitive growth (Black \& Aboud, 2011).

In particular, a large body of research highlights the importance of caregiver sensitivity and responsiveness during feeding as a foundation for high quality interactions that promote infants' abilities to self-regulate intake (DiSantis, Hodges, Johnson, \& Fisher, 2011) and achievement of positive developmental outcomes (Engle \& Pelto, 2011; Farrow \& Blissett, 2014; Hurley, Cross, \& Hughes, 2011; Paul et al., 2016; Savage, Birch, Marini, Anzman-Frasca, \& Paul, 2016). During early infancy, when self-regulatory capacities are low, caregivers can serve as an external source of regulation, helping infants determine appropriate ways to recognize and respond to discomforts, such as hunger and fullness, tiredness, pain, and fear. Sensitivity and contingent responsiveness are typically seen in the form of synchrony of infant and maternal affect and behaviors. In particular, what appears to be important for infants' development of effective self-regulation skills is that the dyad experiences appropriate reparation when there is discord (Tronick, Als, Adamson, Wise, \& Brazelton, 1978); this repeated experience of experiencing discord (e.g., the infant's discomfort due to hunger or tiredness) and appropriate reparation to a more comfortable state (e.g., being fed or soothed to sleep, respectively) helps the infant learn how to regulate his or her own states (Tronick et al., 1977, 1978). Thus, through consistent and repeated exposure to maternal sensitivity and contingent responsiveness, infants eventually learn to self-regulate, as evidenced by the fact that towards the end of infancy, infants show improvements in their abilities to self-soothe and disengage (Berger, Kofman, Livneh, \& Henik, 2007). Specific to feeding outcomes, infants show healthier weight gain trajectories when their mothers use more sensitive and child-centered feeding and parenting approaches (Farrow \& Blissett, 2006; Savage et al., 2016). Additionally, lower maternal sensitivity to infants' cues is predictive of greater infant weight gain in older, but not younger, infants, potentially indicating that poor intake regulation is a learned response that develops over time and is reinforced through low sensitivity to feeding cues (Worobey, Lopez, \& 
Hoffman, 2009). In light of this body of research, the Institute of Medicine (IOM) (Institute of Medicine, 2011) and others (Lumeng, Taveras, Birch, \& Yanovski, 2015) have identified responsive feeding as a critical area in need of further study.

A potential barrier to today's caregivers' abilities to fully attend to their infant during caregiver-infant interactions is the omnipresence of portable technology, such as smart phones and tablets, that facilitate the consumption of digital media at virtually any time (Pew Research Center, 2014). Although limited population data on caregivers' use of technological devices and engagement with digital media during infant feeding and care interactions are available, a previous study demonstrated technological device use during feeding was a common occurrence for mothers in the study, with $83 \%$ reporting engaging with some type of technological device (e.g., watching television or using a mobile device) during infant feeding (Golen \& Ventura, 2015).

Experimental research with parents of older children illustrate effects of technological device use on parent-child interaction quality and child outcomes. When parents and children interact in the presence of a television set playing an adult-directed program ("background television"), parents talk to their children less and use lower-quality speech patterns compared to when the television is turned off (Kirkorian, Pempek, Murphy, Schmidt, \& Anderson, 2009; Pempek, Kirkorian, \& Anderson, 2014; Tanimura, Okuma, \& Kyoshima, 2007). Additionally, background television decreases children's engagement and focused attention while playing with toys (Schmidt, Pempek, Lund, \& Anderson, 2008) and children's learning of new words is hindered when their mothers' attempts to teach new words is interrupted by a cell phone call (Reed, Hirsh-Pasek, \& Golinkoff, 2017).

To date, no studies have similarly studied maternal technological device or digital media use during early feeding interactions within controlled, experimental settings. Previous observational research suggests that mothers who were distracted during a laboratory-based feeding observation showed lower sensitivity to their infants' cues compared to mothers who are not distracted (Golen \& Ventura, 2015b), but the observational nature of these data make it unclear whether mothers with initially low levels of sensitivity to their children choose to engage with a technological device during feeding interactions, or whether the use of a technological device directly lowers the quality of the feeding interaction. To this end, the primary objective of the present within-subject, experimental study was to explore whether mothers' digital media use (that is, watching a television show on a small, portable tablet) during infant feeding affects the quality of maternal-infant dyadic interaction (which includes both the mother's sensitivity and responsiveness to infant cues and engagement in socioemotional and cognitive growth fostering and the infant's clarity of cues or responsiveness to the mother). Because it is feasible that the quality of the dyadic interaction during feeding may impact the infant's feeding behaviors, potential impacts of digital media use during feeding on infant feeding behaviors (e.g., intake, feed duration, rate of feeding) were also examined. Additionally, given mother-infant dyads come to the laboratory with a shared history of experiences that shape their feeding behaviors and interaction styles, which may include typical use of technological devices during feeding interactions, a secondary objective of this study was to explore whether mothers' self-reported technological device use during feeding modified associations between digital media use during feeding and the quality of maternal-infant dyadic interaction or infant feeding behaviors. Based on the limited previous research on this topic, it was hypothesized that mothers would exhibit lower sensitivity and responsiveness to infant cues and less engagement in socioemotional and cognitive growth fostering when they used digital media during feeding compared to when they did not and that infants would similarly show altered feeding behaviors indicative of distracted feeding (e.g., lower intake, longer feed duration, slower rate of feeding).

\section{Method}

\subsection{Participants}

Mothers $(n=25)$ with healthy, term infants of either sex participated in this study. Inclusion criteria for infants were: (1) healthy; (2) born full-term; (3) 32 weeks of age or younger (and, thus, still predominantly milk-fed (Grummer-Strawn, Scanlon, \& Fein, 2008)). To control for possible effects of feeding mode (directly from the breast versus from a bottle) on the quality and outcome of the feeding interaction, only breastfeeding infants were included. Inclusion criteria for mothers included: (1) between 18 and 40 years of age and (2) absence of any complications during pregnancy or birth that lead to infant feeding problems. Participants were recruited through fliers posted in Special Supplemental Nutrition Program for Women, Infants, and Children (WIC) offices, breastfeeding support groups, libraries, coffee shops, and local pediatric offices, as well as through targeted Facebook advertisements. Mothers were blinded to study objectives and hypotheses and were only told that the purpose of the study and videorecorded feeding observations was to "better understand infant feeding behaviors during typical feeding interactions." Mothers received a onesie and book for their infant as compensation for study participation. All study procedures were approved by the university Institutional Review Board and informed consent was obtained from each mother at study entry.

\subsection{Experimental design}

Based on a within-subject design wherein each dyad served as its own control, mother-infant dyads visited our laboratory on two separate days for feeding observations; each laboratory visit occurred at the same time of day to control for infants' circadian rhythms and variation in intake and behavior (Thomas, 1995) and, on average, laboratory visits were separated by $2.4 \pm 1.8$ days. For these feeding observations, mothers were asked to feed their infants under one experimental and one control condition; order of conditions was counterbalanced. During both conditions, mothers breastfed their infants.

(1) During the Digital Media Use condition, mothers were asked to watch a 22-min long TV show on a small tablet (Apple iPad Air [Apple Inc., Cupertino, CA]) while they fed their infants. Mothers chose among four preselected episodes of popular sitcoms (The Office, Friends, Parks and Rec, or Modern Family); each episode was prescreened to ensure content was similar in duration and valence and free of commercials, violence or sexual content, and references to infants, infant feeding, and eating. Previous research has shown that young infants feed for approximately $10 \mathrm{~min}$ (Golen \& Ventura, 2015; Golen \& Ventura, 2015b; Ventura et al., 2012; Ventura, Inamdar, \& Mennella, 2015); thus, it was expected that a single episode would last the duration of the feeding. If needed, a second episode of each sitcom was available to play immediately after the first. Digital media use was stopped when the mother indicated she was done feeding her infant.

(2) During the Control condition, mothers were asked to listen to Rachmaninoff's Second Symphony while feeding their infants. The music was played at a volume of $40 \mathrm{~dB}$, which is considered to be the volume of ambient noise (Mehta, Zhu, \& Cheema, 2012). This classical music played at ambient-levels was used as the control condition because previous research examining the influence of technological devices on adults' eating behaviors has determined that listening to classical music is preferable to silence to help prevent the discomfort of boredom (Blass et al., 2006). This symphony is approximately 66-min in length, but was turned off when the mother indicated she was done feeding her infant. The room was free of all other potential technological or non-technological distractions. 


\subsection{Protocol and measures}

During the three days prior to and throughout the experimental period, mothers were asked to refrain from introducing additional foods or liquids to their infants to minimize atypical infant behavior due to negative reactions. To encourage adherence, mothers kept a daily record of when and what they fed their infants. Within this daily record, mothers also indicated what else, if anything, they did while feeding their infants to provide a sense of typical feeding patterns and levels of technological device use during feeding.

\subsubsection{Feeding Observations}

For the feeding observation, the entire feeding session was videorecorded using a Canon VIXIA HF M41 Full HD Camcorder (Canon, New York, USA). The video camera was placed at the far corner of the room, approximately 10-12 feet from the dyad. The experimenter was concealed behind a partition during the feeding to minimize influence on the feeding interaction. Infant intake was assessed by weighing the infant before and after the feeding using an infant scale (model 374; Seca, Hamburg, Germany). To ensure intake assessments were not affected by changes in clothing or diaper weight, research assistants ensured infants were weighed in the exact same clothes and diaper during the pre- and post-feeding weighing. Intake $(\mathrm{g})$ was converted to volume (mL) assuming a breast milk density of $1.03 \mathrm{~g} / \mathrm{mL}$ (Riordan, 2005). Duration of feeding was measured in minutes and defined as the time between the first instance that the infant latched onto the breast and the mother's verbal indication that the feeding was over. Additionally, rate of feeding $(\mathrm{mL} / \mathrm{min})$ was calculated by dividing intake $(\mathrm{mL})$ by duration of feeding (min). To assess maternal enjoyment and engagement during each condition, mothers were asked "Did you like the music/show?" (response options: Yes, No, Neutral, I don't know) at the end of each feeding.

\subsubsection{Objective Assessment of the Quality of the Maternal-Infant Dyadic Interaction}

After each visit, two trained coders unaware of the experimental conditions and hypotheses of the study scored the video-records using the Nursing Child Assessment Parent-Child Interaction Feeding Scale (NCAFS) (Oxford and Findlay, 2015). This scale has been validated for assessment of the quality of early feeding interactions (breastfeeding, bottle-feeding, or solid food-feeding) for mothers and their infants aged $<12$ months during both laboratory- and home-based feeding observations. This scale is comprised of 76 observable behaviors that are organized into six subscales, four of which describe the mother's contributions to the feeding interaction and two of which describe the infant's contribution (Oxford and Findlay, 2015). Maternal subscales include Sensitivity to Cues (example item: "Caregiver terminates the feeding when the child shows satiation cues or after other methods have proved unsuccessful"; possible score range $=0-16$ with higher scores indicating greater sensitivity to infant cues), Response to Child's Distress (example item: "When infant exhibits a potent disengagement cue, the caregiver stops or starts the feeding"; possible score range $=0-11$ with higher scores indicating greater contingent responsiveness to infant distress), Social-Emotional Growth Fostering (example item: "Caregiver uses positive statements when talking to the child during the feeding"; possible score range $=0-14$ with higher scores indicating greater engagement in behaviors that support infant social-emotional development), and Cognitive Growth Fostering (example item: "Caregiver talks to the child using two words at least three times during the feeding"; possible score range $=0-9$ with higher scores indicating greater engagement in behaviors that support infant cognitive development). Infant subscales include Clarity of Cues (example item: "Child demonstrates satiation at end of feeding"; possible score range $=0-15$ with higher scores indicating greater clarity of cues) and Responsiveness to Caregiver (example item: "Child vocalizes to the caregiver during feeding"; possible score range $=0-11$ with higher scores indicating greater engagement with and responsiveness to the caregiver). Prior to coding, raters were trained by a certified NCAFS trainer and trained to $85 \%$ reliability using the NCAFS training standards (Oxford and Findlay, 2015). After training was complete, study-specific inter-rater reliability was assessed by common-coding of 10 study videos and intrarater reliability was determined by double-coding of 5 study videos. Inter- and intra-rater reliability were established using Pearson's correlation coefficients, and were $r=0.89$ and $r=0.95$, respectively.

\subsubsection{Assessment of Maternal Demographics}

At the end of the second visit day, mothers completed a family demographic questionnaire to assess maternal sociodemographic characteristics and parity.

\subsection{Data analysis}

Using methods previously described (Golen \& Ventura, 2015; Ventura \& Teitelbaum, 2017), we conducted a qualitative analysis of feeding records kept by mothers during the 3-days prior to study participation to obtain a measure of mothers' typical levels of technological device use during feeding (defined as digital media or internet use on a cellphone, tablet, computer, or television). We then determined, for each mother, the percentage of feedings during which use of a technological device was reported $(=$ number of feedings wherein use of a technological device was reported/total number of feedings reported] *100). Median splits were used to classify mothers as: (1) typically low technological device use versus (2) typically high technological device use.

All quantitative analyses were conducted using SAS v.9.4 (SAS Institute Inc., North Carolina, USA). Data were thoroughly cleaned and assessed for normality prior to analysis. Preliminary analyses revealed that the following NCAFS subscales were negatively skewed: maternal sensitivity to infant cues, responsiveness to infant distress, and socioemotional growth fostering, and infant clarity of cues and responsiveness to caregiver; thus, these subscales were normalized using log transformations prior to analyses. After analyses, data were backtransformed by calculating the antilog of the estimate (Bland \& Altman, 1996).

Repeated-measures Analysis of Variance (RM-ANOVA) using SAS PROC MIXED was used to assess the effect of condition (i.e., Digital Media Use versus Control) on the following behaviors and outcomes: (1) mothers' sensitivity to cues, response to distress, social-emotional growth fostering, and cognitive growth fostering; (2) infants' clarity of cues and responsiveness to caregiver; and (3) infant intake (mL), duration of feeding $(\mathrm{min})$, and rate of feeding $(\mathrm{mL} / \mathrm{min})$. To ensure other aspects of testing, such as testing day (first versus second) or order of condition presentation (Digital Media Use then Control versus Control then Digital Media Use) did not impact feeding outcomes, separate RM-ANOVA models were conducted with testing day and condition order as predictor variables. Mothers' typical level of technological device use was tested as a possible moderator of the effects of digital media use on feeding behaviors and outcomes. To this end, mothers' typical level of technological device use (typically low versus typically high) was included in the model testing the effect of condition on feeding behaviors and outcomes. Moderation was determined by a significant interaction between mothers' typical level of technological device use and condition.

Infant age was covaried in all analyses. Models examining outcomes related to infant feeding (infant intake, duration of feeding, or rate of feeding) were also covaried for the amount of time elapsed since the last feeding (prior to the infants' arrival to our laboratory, as reported by mothers). A $p$-value $<.05$ was used as a criterion for statistical significance of main and interaction effects; statistical trends were also noted and defined as $p<.10$. 


\section{Results}

\subsection{Sample characteristics}

Fourteen (56\%) of infants were female and average infant age was $19.3 \pm 6.4$ weeks (range $=6.2-32.0$ weeks). The majority of infants were exclusively breastfed, with only 2 infants receiving a mix of breast milk and formula. Mothers were $31.2 \pm 3.4$ years old (range $=25.3-40.0$ years) and $76 \%(n=19)$ were primiparous. The majority of mothers reported an annual family income $\geq \$ 75,000$ $(72 \%, n=18)$, held a bachelors or graduate degree $(68 \%, n=17)$, were married $(92 \%, n=23)$ and identified as non-Hispanic white $(80 \%, n=20)$.

\subsection{Associations between digital media use, maternal-infant dyadic interaction quality, and infant feeding behaviors}

No significant effects of testing day or order of condition presentation were seen for any feeding behavior or outcome (all $p \geq .05$ ). Eighty-eight percent $(n=22)$ of mothers reported that they enjoyed the show they watched during the Digital Media Use condition, 2 mothers indicated they felt neutral about the show, and 1 mother indicated that she did not like the show. Similarly, $84 \%(n=21)$ of mothers indicated they liked the music that they listened to during the Control condition, 3 mothers indicated they felt neutral about the music, and 1 mother indicated she did not like the music.

As illustrated in Table 1, there was a trend for mothers to show lower sensitivity to infant cues during the Digital Media Use condition compared to the Control condition $(p=.098)$. There was also a significant effect of condition on cognitive growth fostering $(p=.018)$ indicating that mothers engaged their infants in significantly less cognitive growth fostering when they used digital media versus when they did not. No effect of condition was seen on any other feeding behavior or outcome.

When mothers' typical level of technological device use was tested as a moderator of the effect of condition on maternal-infant dyadic interaction quality and infant feeding behaviors, a significant interaction was seen when considering infant responsiveness to the mother

Table 1

Effects of maternal digital media use during feeding on maternal-infant dyadic interaction quality and infant feeding behaviors $(\mathrm{N}=25)$.

\begin{tabular}{|c|c|c|c|c|}
\hline & Control $^{\mathrm{a}}$ & $\begin{array}{l}\text { Digital Media } \\
\text { Use }^{a}\end{array}$ & $F$-value & $p$-value \\
\hline \multicolumn{5}{|l|}{ Maternal NCAFS Subscales } \\
\hline Sensitivity to Infant Cues ${ }^{b, c}$ & $14.8(0.3)$ & $14.4(0.3)$ & 3.01 & .098 \\
\hline $\begin{array}{l}\text { Responsiveness to Infant } \\
\text { Distress }\end{array}$ & $10.0(0.3)$ & $10.1(0.3)$ & 0.12 & .732 \\
\hline $\begin{array}{l}\text { Social-Emotional Growth } \\
\text { Fostering b,e }\end{array}$ & $10.9(0.5)$ & $10.6(0.5)$ & 0.57 & .457 \\
\hline $\begin{array}{l}\text { Cognitive Growth Fostering b,f } \\
\text { Infant NCAFS Subscales }\end{array}$ & $5.8(0.4)$ & $5.1(0.4)$ & 6.62 & .018 \\
\hline Clarity of Cues ${ }^{b, g}$ & $12.1(0.3)$ & $11.6(0.3)$ & 1.40 & .250 \\
\hline Responsiveness to Caregiver ${ }^{\mathrm{b}, \mathrm{d}}$ & $6.3(0.5)$ & $5.5(0.5)$ & 2.19 & .154 \\
\hline \multicolumn{5}{|c|}{ Infant Intake and Feeding Behaviors } \\
\hline Intake $(\mathrm{mL})^{\mathrm{h}}$ & $95.2(15.2)$ & $99.2(15.2)$ & 0.05 & .832 \\
\hline Feed duration (min) ${ }^{h}$ & $15.3(1.3)$ & $14.7(1.3)$ & 0.58 & .452 \\
\hline Feed rate $(\mathrm{mL} / \mathrm{min})^{\mathrm{h}}$ & $7.7(1.5)$ & $8.5(1.5)$ & 0.25 & .622 \\
\hline
\end{tabular}

NCAFS, Nursing Child Assessment Parent-Child Interaction Feeding Scale.

a Column values are Mean (SE).

b Model adjusted for infant age.

c Possible score range $=0-16$.

d Possible score range $=0-11$.

e Possible score range $=0-14$.

f Possible score range $=0-9$.

g Possible score range $=0-15$.

h Model adjusted for infant age and time since last feeding.

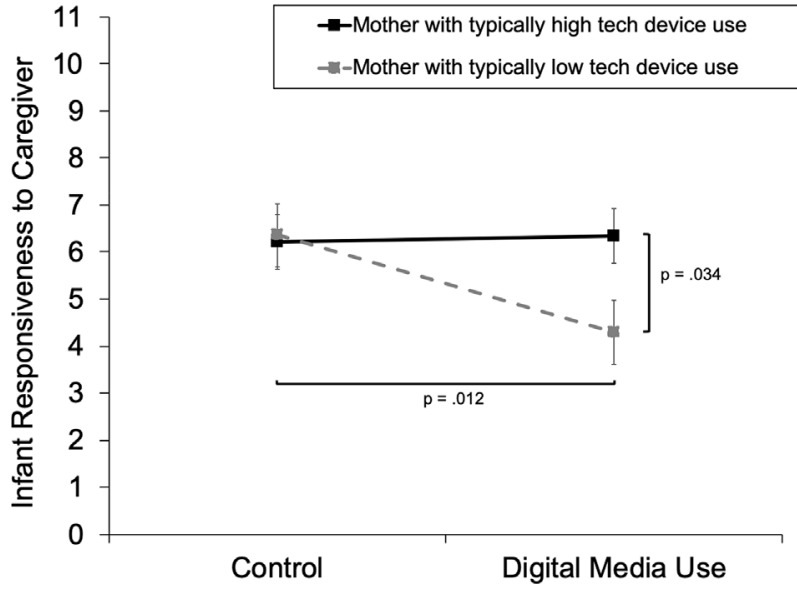

Fig. 1. Mothers' typical level of technological device use moderated the effect of maternal digital media use during feeding on infant responsiveness to caregiver. Post hoc analyses revealed that when mothers' typical level of technological device use during feeding was low, infants showed a significant decrease in their responsiveness to their mother during the Digital Media Use condition compared to the Control condition $(p=.012)$. Additionally, infants of mothers with typically low levels of technological device use during feeding were significantly less responsive to their mother during the Digital Media Use condition compared to infants of mothers with typically high levels of technological device use $(p=.034)$.

( $p=.033$ ). As illustrated in Fig. 1, when mothers' typical level of technological device use was low, infants showed a significant decrease in their responsiveness to their mother during the Digital Media Use condition compared to the Control condition $(p=.012)$. Infants of mothers with typically low levels of technological device use were significantly less responsive to their mother during the Digital Media Use condition compared to infants of mothers with typically high levels of technological device use $(p=.034)$.

\section{Discussion}

The present study was, to our knowledge, the first to experimentally explore associations between maternal digital media use (defined here as watching a television show on a tablet) during feeding and maternalinfant dyadic interaction quality and infant feeding behaviors. This research provides a foundation for further research aimed at understanding maternal technological device use during infant feeding interactions. This study overcame some limitations of previous research examining associations between technological device use and child outcomes due to the strengths of our within-subject, experimental approach, which include abilities to control the type of technological device mothers were exposed to and to eliminate many of the biases inherent to self-reported data and self-selected behaviors. Using this approach, two key findings emerged. First, we found effects of digital media use on some aspects of the quality of feeding interactions, as indicated by a trend toward an effect of digital media use on lowering maternal sensitivity to infant cues and a significant effect of digital media use on lowering mothers' engagement in cognitive growth fostering with their infants. Second, we found that infants' response to maternal digital media use depended on how typical maternal technological device use during feeding was for the infant. In particular, infants of mothers with typically low levels of technological device use during feeding exhibited lower responsiveness to their mothers during the Digital Media Use compared to Control condition, whereas infants of mothers with typically high levels of technological device use during feeding showed no behavioral changes across the experimental conditions.

Given the ubiquity of television, mobile devices, and handheld 
technologies (Pew Research Center, 2014), it is unsurprising that significant proportions of mothers report engaging with technological devices while feeding their infants (Golen \& Ventura, 2015; Ventura \& Teitelbaum, 2017). In response, the popular press and lay-media are filled with ominous warnings about the dangers of "brexting" (breastfeeding and texting) and other forms of technology use during motherinfant interactions, despite a scant evidence-base regarding whether we should be worried about mothers' occasional or habitual use of digital media during infant feeding interactions. Of primary concern is the potential for maternal digital media use to decrease beneficial features of caregiver-child interactions that have been implicated in positive developmental outcomes. For example, the importance of caregivers' sensitivity and contingent responsiveness to infant cues within both feeding and non-feeding contexts has been well-established (Gunning, Halligan, \& Murray, 2013; Harrist \& Waugh, 2002; Isabella \& Belsky, 1991; Reyna \& Pickler, 2009; Tronick et al., 1978; Yoo \& ReebSutherland, 2013), and maternal non-responsiveness is a significant stressor for infants with consequences for infants' developing abilities to self-regulate (Moore \& Calkins, 2004; Moore et al., 2009). In light of this knowledge, the possibility that maternal digital media use decreases maternal sensitivity to infant cues supports the need for further research on this topic. That the effect of digital media use on maternal sensitivity to infant cues approached, but did not reach, significance may suggest that individual variability exists in how distracted mothers are by digital media during feeding, with some mothers fully absorbing in the technology and others periodically checking in with and attending to their infants despite the presence of digital media. In the present study, we did not include a direct measure of mothers' attention to the digital media versus the infant during the feeding, which would have provided additional, valuable information about how distracted mothers were during the feeding. Thus, further research with additional measures and larger samples is needed to better understand potential individual differences in how mothers interact with technological devices versus their infants during feeding interactions and what implications these interactions have for mothers' abilities to be sensitive and responsive to their infants' feeding cues.

Cognitive growth fostering during feeding interactions involves actions such as maternal encouragement of infant exploration and learning, engagement of the infant in cognitively stimulating ways, and use of infant-directed speech aimed at describing the food, aspects of the feeding, or other things going on during the feeding (Oxford and Findlay, 2015). Our finding that digital media use during feeding led to significantly less cognitive growth fostering is concerning given the importance of these behaviors for infants' developing language and cognitive abilities (Bornstein and Tamis-LeMonda, 1989; ClarkeStewart, 1973; Lewis, 1993; Olson, Bates, \& Bayles, 1984; Vygotsky, 1978), and is consistent with previous research illustrating technological devices decrease parents' use of child-directed speech during interactions with older children (Kirkorian et al., 2009; Pempek et al., 2014; Tanimura et al., 2007). Given benefits of caregivers' use of highquality, infant-directed speech on infants' language and cognitive development (Saint-Georges et al., 2013), it is possible that mothers' habitual use of technological devices during feeding could lead to poorer language and cognitive development for infants, but further longitudinal research is needed.

Our measure of infants' responsiveness to their mothers encompassed actions such as responding to the mothers' feeding attempts, games, and social cues and engaging in mother-directed looking, smiling, and vocalizing (Oxford and Findlay, 2015); thus, infant responsiveness to the mother during feeding is an important part of maternal-infant synchrony that creates a high-quality and engaging interactions. The present study suggests the presence of a technological device during feeding may decrease some infants' willingness or ability to reciprocate to mothers' attempts at social interaction during feeding. Additionally, that infants' previous history with maternal technological device use during feeding moderated effects of maternal digital media use on infant behavior may represent infant adaptation or habituation to technological device use during feeding (Kaplan \& Werner, 1986; Kavsek, 2013). However, it is also possible that infants' reactions to the presence of technological devices during feeding are a driver or facilitator of maternal technological device use during feeding. Maternal perceptions of infant responses to aspects of the food or feeding interaction can drive maternal perceptions and feeding behaviors (Cartagena et al., 2014; Forestell \& Mennella, 2012), thus mothers of infants who appear unaffected by the presence of a technological device during feeding may be more apt to continue using that technology. Conversely, if mothers notice that their infant is distracted during feedings where a technological device is present, the mothers may be less inclined to use technological devices during feeding interactions. We did not collect data on mothers' motivations for or against using technological devices during feeding, but this would be an important area of further research, as well as longitudinal research looking at long-term changes in infant behavior when mothers increase or decrease their use of technological devices during feeding.

Overall, this study was a first step toward understanding maternal digital media use during feeding; however, this study is not without limitations. The media to which mothers were exposed in the experimental condition was preselected to standardize the valence and content of the digital media exposure across participants; this level of control can be viewed as a strength of the experimental design but it is also possible that mothers would have been more engaged with media that they had self-selected or with a different type of media, such as an interactive or social media app. Similarly, in-line with previous research examining effects of television and digital media use on adults' eating behaviors (e.g. (Blass et al., 2006)), we elected to have mothers listen to neutral, classical music played at ambient-levels during the control condition to prevent the potential discomfort of a completely stimulus-free environment (which may have been unnatural for most mothers); however, it is possible that this control condition was unfamiliar to mother-infant dyads and thereby distracting in and of itself. Given that a subset of mothers reported high technology use during feeding, it is also possible that the control condition was especially abnormal or uncomfortable for these mothers. Future research that also includes an unmanipulated feeding observation within a familiar environment (e.g., the dyad's home) may provide additional context and insights for understand the dyad's interaction within a manipulated laboratory setting.

It should also be noted that cognitive growth fostering scores in this study were $\sim 1$ point lower than published scores from the NCAFS Database (Oxford and Findlay, 2015); within this database of 1638 mother-infant dyads (infant age range: 0 - 12 months), mean cognitive growth fostering scores were $6.6 \pm 2.1$ compared to $5.4 \pm 1.8$ in the present study. Given previous research illustrating cognitive growth fostering scores are significantly and positively correlated with infant age $(r=.31, p<.05)$ (Oxford and Findlay, 2015), one possible explanation for this discrepancy is the lower age range of the infants in the present study (1.5-7 months) compared to the reference database. In addition to being limited to mothers of young infants, this small sample consisted of predominantly non-Hispanic white, middle- to upper-socioeconomic status mothers who were breastfeeding. We did not include a measure of maternal psychopathological risk (e.g., postpartum depression), which is an important risk factor for impaired sensitivity and responsiveness (e.g. (Hurley, Black, Papas, \& Caulfield, 2008; Savage \& Birch, 2017; Tronick \& Reck, 2009)) and, thus, may be an important risk factor for media use during feeding or effects of digital media use on the quality and outcome of feeding interactions. In sum, further research with larger, more diverse samples and additional measures of maternal and infant characteristics and behaviors is needed.

It is important to note that there are potential benefits of mothers' technology use that should not be overlooked. Previous research suggests that mothers view the availability of social networking 
technologies (e.g., blogs, Facebook) as an important source of social connection, information, and advice (McDaniel, Coyne, \& Holmes, 2012). Technological device use can also provide mothers and other caregivers with important opportunities to "take a break" from their caregiving duties and recharge during times when children are not biding for a caregiver's attention (Radesky et al., 2016). Thus, when used mindfully and in moderation, it may be possible for caregivers to effectively balance the benefits of technology use with the benefits of engaging their child in high quality interactions.

In addition, within the present study, infant intake, meal duration, and rate of feeding were unaffected by maternal digital media use, which may suggest that digital media use does not interfere with the nutritive aspects of infant feeding interactions. If further research with larger, more diverse samples confirms the findings of the present study, it is possible that maternal digital media use during feeding is not a behavior that should be discouraged, particularly if it increases the likelihood that a mother would continue breastfeeding. Further research in this field should continue to weigh the potential positive and/ or negative impacts of the ubiquity of portable technology on caregivers, children, and the quality and outcome of caregiver-child interactions.

\section{Conclusions}

The present study explored associations between maternal digital media use and the quality of infant feeding interactions and highlights avenues for further research. This research illustrated maternal digital media use during feeding affected some aspects of the feeding interactions in the short-term, but other aspects of the feeding interaction were unchanged. Future studies evaluating maternal digital media use during feeding and infant feeding outcomes should, first and foremost, include larger sample sizes, as well as more diverse samples to improve the generalizability of findings. In addition to further quantitative research on associations between digital media use and feeding outcomes, it would be useful to have more qualitative research examining mothers' motivations for and perceptions of digital media use during feeding and other aspects of infant care, as well as how digital media use may vary throughout the day (e.g., during the day versus the middle of the night). This research would provide greater insights into how to support mothers' needs for potential benefits provided by digital media use during feeding, while also providing targeted guidance for how to balance digital media use with attention to infants' cues and needs during feeding and other infant care interactions. The potential impact of maternal digital media use on both short-term and long-term feeding and developmental outcomes is an important question for future inquiry and may lead to novel education efforts to improve maternal sensitivity and responsiveness, as well as the quality of maternal-infant interactions, during early feeding.

\section{Source of funding}

Simms/Mann Institute for Education and Community Development.

\section{Conflicts of interest}

The authors have no conflicts of interest relevant to this article to disclose.

\section{Acknowledgements}

This project was supported by the Simms/Mann Institute for Education and Community Development. We thank the mothers and infants who participated in this study. We also thank Brittany Lore, Claire Gleadhill, Teresa Sanchez, Megan Breadmore, Simone Teitelbaum, and Alexandra Hernandez for their technical assistance.

\section{Appendix A. Supplementary data}

Supplementary data to this article can be found online at https:// doi.org/10.1016/j.appet.2019.104415.

\section{References}

Berger, A., Kofman, O., Livneh, U., \& Henik, A. (2007). Multidisciplinary perspectives on attention and the development of self-regulation. Progress in Neurobiology, 82(5), $256-286$.

Black, M. M., \& Aboud, F. E. (2011). Responsive feeding is embedded in a theoretical framework of responsive parenting. Journal of Nutrition, 141(3), 490-494.

Bland, J. M., \& Altman, D. G. (1996). Transforming data. British Medical Journal, 312(7033), 770.

Blass, E. M., Anderson, D. R., Kirkorian, H. L., Pempek, T. A., Price, I., \& Koleini, M. F. (2006). On the road to obesity: Television viewing increases intake of high-density foods. Physiology \& Behavior, 88(4-5), 597-604.

Bornstein, M. H., \& Tamis-LeMonda, C. S. (1989). Maternal responsiveness and cognitive development in children. In M. H. Bornstein (Ed.). Maternal responsiveness: Characteristics and consequences (pp. 49-61). San Fransico: Jossey-Bass.

Cartagena, D. C., Ameringer, S. W., McGrath, J., Jallo, N., Masho, S. W., \& Myers, B. J. (2014). Factors contributing to infant overfeeding with Hispanic mothers. Journal of Obstetric, Gynecologic, and Neonatal Nursing, 43(2), 139-159.

Clarke-Stewart, K. A. (1973). Interactions between mothers and their young children: Characteristics and consequences. Monographs of the Society for Research in Child Development, 38 Serial No. 153.

DiSantis, K. I., Hodges, E. A., Johnson, S. L., \& Fisher, J. O. (2011). The role of responsive feeding in overweight during infancy and toddlerhood: A systematic review. International Journal of Obesity, 35(4), 480-492.

Engle, P. L., \& Pelto, G. H. (2011). Responsive feeding: Implications for policy and program implementation. Journal of Nutrition, 141(3), 508-511.

Farrow, C., \& Blissett, J. (2006). Does maternal control during feeding moderate early infant weight gain? Pediatrics, 118(2), e293-298.

Farrow, C., \& Blissett, J. (2014). Maternal mind-mindedness during infancy, general parenting sensitivity and observed child feeding behavior: A longitudinal study. Attachment \& Human Development, 16(3), 230-241.

Fomon, S. J. (1993). Nutrition of normal infants. St. Louis, MO: Mosby-Year Book, Inc.

Forestell, C. A., \& Mennella, J. A. (2012). More than just a pretty face. The relationship between infant's temperament, food acceptance, and mothers' perceptions of their enjoyment of food. Appetite, 58(3), 1136-1142.

Golen, R. B., \& Ventura, A. K. (2015a). What are mothers doing while bottle-feeding their infants? Exploring the prevalence of maternal distraction during bottle-feeding interactions. Early Human Development, 91(12), 787-791.

Golen, R. B., \& Ventura, A. K. (2015b). Mindless feeding. Is maternal distraction during bottle-feeding associated with overfeeding? Appetite, 91, 385-392.

Grummer-Strawn, L. M., Scanlon, K. S., \& Fein, S. B. (2008). Infant feeding and feeding transitions during the first year of life. Pediatrics, 122(Suppl 2), S36-S42.

Gunning, M., Halligan, S. L., \& Murray, L. (2013). Contributions of maternal and infant factors to infant responding to the still face paradigm: A longitudinal study. Infant Behavior and Development, 36(3), 319-328.

Harrist, A. W., \& Waugh, R. M. (2002). Dyadic synchrony: Its structure and function in children's development. Developmental Review, 22(4), 555-592.

Hurley, K. M., Black, M. M., Papas, M. A., \& Caulfield, L. E. (2008). Maternal symptoms of stress, depression, and anxiety are related to nonresponsive feeding styles in a statewide sample of WIC participants. Journal of Nutrition, 138(4), 799-805.

Hurley, K. M., Cross, M. B., \& Hughes, S. O. (2011). A systematic review of responsive feeding and child obesity in high-income countries. Journal of Nutrition, 141(3), 495-501.

Institute of Medicine (2011). Early childhood obesity prevention policies. Washington, D.C.: National Academies Press.

Isabella, R. A., \& Belsky, J. (1991). Interactional synchrony and the origins of infantmother attachment: A replication study. Child Development, 62(2), 373-384.

Kaplan, P. S., \& Werner, J. S. (1986). Habituation, response to novelty, and dishabituation in human infants: Tests of a dual-process theory of visual attention. Journal of Experimental Child Psychology, 42(2), 199-217.

Kavsek, M. (2013). The comparator model of infant visual habituation and dishabituation: Recent insights. Developmental Psychobiology, 55(8), 793-808.

Kirkorian, H. L., Pempek, T. A., Murphy, L. A., Schmidt, M. E., \& Anderson, D. R. (2009). The impact of background television on parent-child interaction. Child Development, 80(5), 1350-1359.

Lewis, M. D. (1993). Early socioemotional predictors of cognitive competency at 4 years. Developmental Psychology, 29, 1036-1045.

Lumeng, J. C., Taveras, E. M., Birch, L., \& Yanovski, S. Z. (2015). Prevention of obesity in infancy and early childhood: A national institutes of health workshop. JAMA Pediatrics, 169(5), 484-490.

McDaniel, B. T., Coyne, S. M., \& Holmes, E. K. (2012). New mothers and media use: Associations between blogging, social networking, and maternal well-being. Maternal and Child Health Journal, 16(7), 1509-1517.

Mehta, R., Zhu, R., \& Cheema, A. (2012). Is noise always bad? Exploring the effects of ambient noise on creative cognition. Journal of Consumer Research, 39(4), 784-799.

Moore, G. A., \& Calkins, S. D. (2004). Infants' vagal regulation in the still-face paradigm is related to dyadic coordination of mother-infant interaction. Developmental Psychology, 40(6), 1068-1080.

Moore, G. A., Hill-Soderlund, A. L., Propper, C. B., Calkins, S. D., Mills-Koonce, W. R., \& 
Cox, M. J. (2009). Mother-infant vagal regulation in the face-to-face still-face paradigm is moderated by maternal sensitivity. Child Development, 80(1), 209-223.

Olson, S. L., Bates, J. E., \& Bayles, K. (1984). Mother-infant interaction and the development of individual differences in children's cognitive competence. Developmental Psychology, 20, 166-179.

Oxford, M., \& Findlay, D. (Eds.). (2015). Caregiver/parent-child interaction: Feeding manual (2nd ed.). Seattle, Washington: NCAST Programs.

Paul, I. M., Savage, J. S., Anzman-Frasca, S., Marini, M. E., Mindell, J. A., \& Birch, L. L. (2016). INSIGHT responsive parenting intervention and infant sleep. Pediatrics, 138(1).

Pempek, T. A., Kirkorian, H. L., \& Anderson, D. R. (2014). The effects of background television on the quantity and quality of child-directed speech by parents. Journal of Children and Media, 8(3), 211-222.

Pew Research Center (2014). Mobile technology fact sheet. http://www.pewinternet.org/ fact-sheets/mobile-technology-fact-sheet/, Accessed date: 27 January 2015.

Radesky, J. S., Kistin, C., Eisenberg, S., et al. (2016). Parent perspectives on their mobile technology use: The excitement and exhaustion of parenting while connected. Journal of Developmental and Behavioral Pediatrics, 37(9), 694-701.

Reed, J., Hirsh-Pasek, K., \& Golinkoff, R. M. (2017). Learning on hold: Cell phones sidetrack parent-child interactions. Developmental Psychology, 53(8), 1428-1436.

Reyna, B. A., \& Pickler, R. H. (2009). Mother-infant synchrony. Journal of Obstetric, Gynecologic, and Neonatal Nursing, 38(4), 470-477.

Riordan, J. (2005). Breastfeeding and human lactation (3rd ed.). Sundbury, MA: Jones and Bartlett Publishers.

Saint-Georges, C., Chetouani, M., Cassel, R., et al. (2013). Motherese in interaction: At the cross-road of emotion and cognition? (A systematic review). PLoS One, 8(10), e78103.

Savage, J. S., \& Birch, L. L. (2017). WIC mothers' depressive symptoms are associated with greater use of feeding to soothe, regardless of perceived child negativity. Pediatric Obesity, 12(2), 155-162.

Savage, J. S., Birch, L. L., Marini, M., Anzman-Frasca, S., \& Paul, I. M. (2016). Effect of the insight responsive parenting intervention on rapid infant weight gain and overweight status at age 1 year: A randomized clinical trial. JAMA Pediatrics, 170(8), 742-749. Schmidt, M. E., Pempek, H. L. K., Lund, A. F., \& Anderson, D. R. (2008). The effects of background television on the toy play behavor of very young children. Child Development, 79(4), 1137-1151.

Tanimura, M., Okuma, K., \& Kyoshima, K. (2007). Televison viewing, reduced parental utteranec, and delayed speech development in infants and young children. Archives of Pediatrics and Adolescent Medicine, 161, 618-619.

Thomas, K. A. (1995). Biorhythms in infants and role of the care environment. Journal of Perinatal and Neonatal Nursing, 9(2), 61-75.

Tronick, E., Als, H., Adamson, L., Wise, S., \& Brazelton, T. B. (1978). The infant's response to entrapment between contradictory messages in face-to-face interaction. Journal of the American Academy of Child Psychiatry, 17(1), 1-13.

Tronick, E. D., Als, H., \& Brazelton, T. B. (1977). Mutuality in mother-infant interaction. Journal of Communication, 27(2), 74-79.

Tronick, E., \& Reck, C. (2009). Infants of depressed mothers. Harvard Review of Psychiatry, 17(2), 147-156.

Ventura, A. K., Beauchamp, G. K., \& Mennella, J. A. (2012). Infant regulation of intake: The effect of free glutamate content in infant formulas. American Journal of Clinical Nutrition, 95(4), 875-881.

Ventura, A. K., Inamdar, L. B., \& Mennella, J. A. (2015). Consistency in infants' behavioural signalling of satiation during bottle-feeding. Pediatric Obesity, 10(3), 180-187.

Ventura, A. K., \& Teitelbaum, S. (2017). Maternal distraction during breast- and bottle feeding among WIC and non-WIC mothers. Journal of Nutrition Education and Behavior, 49(7S2), S169-S176 e161.

Vygotsky, L. S. (1978). Mind in society: The development of higher psychological processes. Cambridge, MA: Harvard University Press.

Worobey, J., Lopez, M. I., \& Hoffman, D. J. (2009). Maternal behavior and infant weigh gain in the first year. Journal of Nutrition Education and Behavior, 41(3), 169-175.

Yoo, K., \& Reeb-Sutherland, B. C. (2013). Effects of negative temperament on 5-monthold infants' behavior during the still-face paradigm. Infant Behavior and Development, 36(3), 344-348. 